ten Patienten mit guter Gewichtsreduktion im ersten Jahr, die von der Therapie profitieren.

Leider ist auch hier schon erkennbar, dass dieser Effekt im Verlauf der vier Jahre deutlich abnehmend ist, gerade wenn man die komplette Remission betrachtet. Zudem ist eine anhaltende Remission über den gesamten Zeitraum nur bei einem deutlich geringeren Teil dieser Patienten vorhanden. Zudem kommt es bekanntermaßen im Verlauf auch in dieser randomisierten Studie zu einer deutliche Gewichtswiederzunahme nach 4 Jahren, trotz anhaltender und umfangreicher Intervention.

Im Vergleich dazu lieferten die Daten der zu einem früheren Erkrankungszeitpunkt einsetzenden finnischen und amerikanischen Diabetespräventionsstudien einen stärkeren Effekt hinsichtlich der Diabetesprävention. Möglicherweise ist demnach bei einmal eingetretener Erkrankung (Typ-2-Diabetes) eine Lebensstilinterventionen deutlich weniger effektiv, um eine Normalisierung der Glukosestoffwechsellage zu erreichen, was den frühen Beginn solcher Maßnahmen weiter unterstützt. Inwieweit diese Daten Auswirkungen auf den primä- ren Endpunkt der Studie haben, bleibt momentan noch abzuwarten. Bekanntermaßen wurde die Studie im September 2012 auf Grund von unzureichender Effektivität hinsichtlich des primären Endpunktes gestoppt.

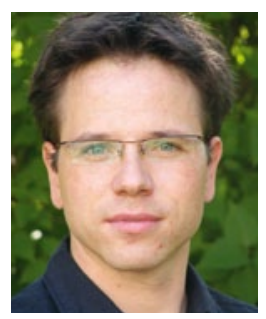

PD Dr. med. Knut Mai

Clinic of Endocrinology, Diabetes and Nutrition Experimental and Clinical Research Center (ECRC) Charite Campus Berlin Buch

Lindenberger Weg 80, 13125 Berlin

und

Charité - Universitätsmedizin Berlin

Campus Mitte

Charitéplatz 1, 10117 Berlin

\title{
Initiative und Forschung für Typ-2-Patienten
}

Remission des Typ-2-Diabetes durch Lebensstil-Intervention: "Wie wurden Sie ein Diabetes-Champion?"

Die Initiative „Diabetes-Champion“ möchte durch eine Öffentlichkeits-Aktion bundesweit möglichst viele Personen finden, die nachweislich an einem Typ-2-Diabetes-mellitus erkrankt waren und durch eine Lebensstiländerung in eine sogenannte Remission gekommen sind. Personen, die sich auf diesen Aufruf bei der Deutschen Diabetes-Stiftung (DDS) melden, werden zunächst gebeten, einen strukturierten Fragebogen auszufüllen. Diese Daten sollen wissenschaftlich ausgewertet werden. In einer weiteren Phase können diese Personen in kooperierenden Diabeteszentren metabolisch charakterisiert werden. Auch sollte dieser Pool an Personen als Vorbilder und Botschafter zur Verfügung stehen.

Aktuelle Veröffentlichungen zeigen, dass in vielen Fällen - durch intensive, nachhaltige Maßnahmen moderater Lebensstil-Änderung (vorwiegend Erhöhung der körperlichen Aktivität sowie ErnährungsUmstellung) - eine Remission des Typ-2-Diabetes realistisch ist (s. a. Beitrag „Dauerhafte Remission manchmal möglich“. Entscheidend ist die Kenntnis der Zusammenhänge einerseits und der Wille des Patienten andererseits - hin zu einer signifikant verbesserten Lebensqualität.

Die Erkenntnis, gut motiviert einen bedeutenden Schritt der Gesunderhaltung erfolgreich bewältigt zu haben, könnte für viele (frisch manifestierte) Diabetiker eine große Erleichterung sein. Der gar nicht so selten - mögliche Verzicht auf orale Antidiabetika und/ oder Insulin ist durch erfolgreiche Umsetzung der Basistherapie die „Chance bei Diabetes".

Bitte unterstützen Sie Ihre interessierten Typ-2-DiabetesPatienten beim Mitmachen bei der Initiative „Werden Sie ein Diabetes-Champion“.
Den Fragebogen und weitere Infos erhalten Sie auf Anfrage bei:

„Chance bei Diabetes" in der DDS

FAX-Nummer: 089 / 579 579-19

eMail: info@diabetesstiftung.de

oder

im Internet unter www.chance-bei-diabetes.de

Initiator und Studienleiter ist Professor Stephan Martin vom Westdeutschen Diabetes- und Gesundheitszentrum in Düsseldorf, Verbund der Katholischen Kliniken Düsseldorf (VKKD)

Falls Sie Fragen zu den Teilnehmerunterlagen oder zum weiteren Vorgehen haben, stehen Ihnen folgende Teams montags bis freitags von 9.00 bis 17.00 Uhr zur Verfügung:

- Deutsche Diabetes-Stiftung (Staffelseestraße 6, 81477 München), Tel. 089579 579-0

- Westdeutsches Diabetes- und Gesundheitszentrum,

Tel. 0211566036065

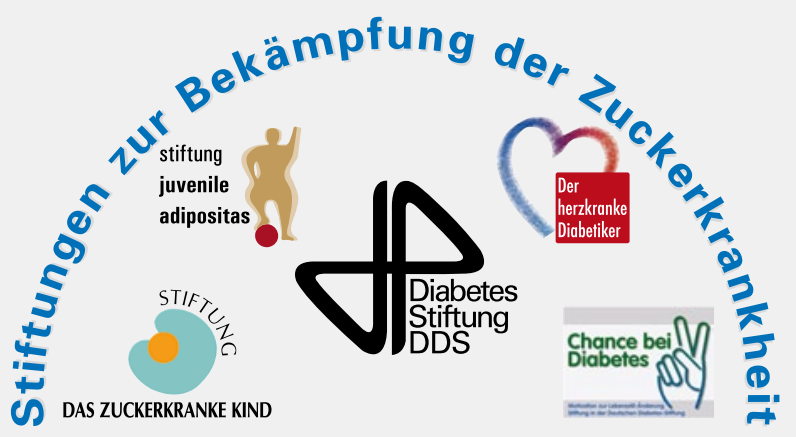

Stiftungen in der DDS - Für die Menschen. Gegen Diabetes. 